\title{
DOCEAMUS
}

doceamus...let us teach

\section{A Missing Piece: Early Elementary Plane Rotations}

\section{Bob Palais}

The formula for plane rotations can and perhaps should be taught at an elementary level, for its usefulness in many fields including geometry, physics, and computer animation, and because it unifies and clarifies a wide range of mathematical subjects.

A rotation of the plane that fixes the origin is determined by its effect on a single reference point. If the image of $(1,0)$ is $(X, Y)$, the plane rotation formula expresses the image of any point $(x, y)$ as the multiple $x$ of

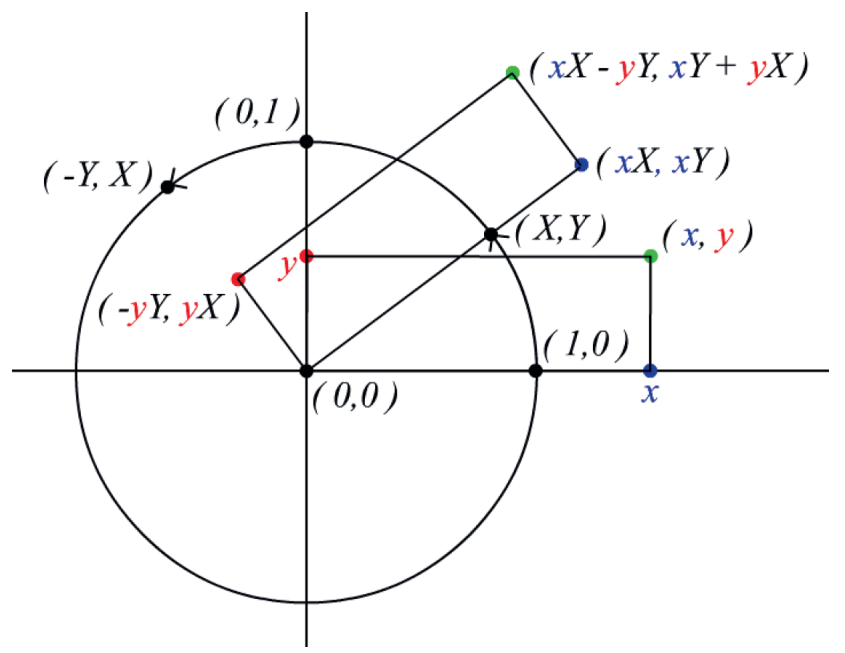

Figure 1 . The plane rotation formula

Bob Palais is associate professor of mathematics at the Utah Valley University. His email address is Bob.Pa1ais@ uvu. edu.

Members of the Editorial Board for Doceamus are: David Bressoud, Roger Howe, Karen King, William McCallum, and Mark Saul.

DOI: http://dx.doi.org/10.1090/noti1079
$(X, Y)$ plus the multiple $y$ of $(-Y, X)$, the image of $(0,1)$ :

(1) $x\langle X, Y\rangle+y\langle-Y, X\rangle=\langle x X-y Y, y X+x Y\rangle$.

The addition and scaling operations call for vector notation $\langle$,$\rangle instead of point no-$ tation ( , ). The Pythagorean relation on $X$ and $Y$ can actually be obtained as a consequence of the formula! The derivation suggested in Figure 1 requires only basic Euclidean geometry. This makes a variety of exciting and useful mathematical topics available much earlier. Organizing the curriculum around this marriage of geometry and algebra can also provide a natural introduction to and excellent preparation for the next levels.

The key to the plane rotation formula is the observation that, when you turn your head $90^{\circ}$ clockwise, what used to look like $(X, Y)$ now looks like $(-Y, X)$. A rotation by a right angle is equivalent to choosing a neighboring pair of perpendicular rays from the axes of the same rectangular system as positive first and second coordinate axes. Applying this twice, the image of $\langle-Y, X\rangle$ is $\langle-X,-Y\rangle$, so a quarter-turn rotation behaves as a square root of -1 . By the same reasoning, if a rotation takes $(1,0)$ to $(X, Y)$, then it must also take $(0,1)$ to $(-Y, X)$. By similar triangle constructions, or simply scaling, the image of $\langle x, 0\rangle$ is $x\langle X, Y\rangle=\langle x X, x Y\rangle$, and the image of $\langle 0, y\rangle$ is $y\langle-Y, X\rangle=\langle-y Y, y X\rangle$. By a congruent triangle construction, or shift of origin, we arrive at (1): the plane rotation that takes $\langle 1,0\rangle$ to $\langle X, Y\rangle$ takes $\langle x, y\rangle$ to $x\langle X, Y\rangle+y\langle-Y, X\rangle$. We will now see how this formula unifies the circular addition formulas, the Pythagorean relation, the 
geometric meanings of complex products, powers, exponentials, the dot and cross products, and more.

With $\langle x, y\rangle=\langle\cos s, \sin s\rangle,\langle X, Y\rangle=\langle\cos t, \sin t\rangle$, (1), the rotation formula (1) becomes

$$
\begin{aligned}
& \langle\cos (s+t), \sin (s+t)\rangle \\
& \quad=\langle\cos s \cos t-\sin s \sin t, \sin s \cos t \\
& \quad+\cos s \sin t\rangle .
\end{aligned}
$$

This reunites the two circular addition formulas that are usually treated separately and better explains their related algebraic structure. In contrast, the kinds of derivations many students experience are like the one given in the 2010 edition of a popular precalculus text. ${ }^{1}$ Starting from a distance formula obtained by unrelated cut and paste methods, $\sqrt{(\cos s-\cos t)^{2}+(\sin s-\sin t)^{2}}$ $=\sqrt{(\cos (s-t)-1)^{2}+(\sin (s-t)-0)^{2}}$, two pages of algebraic manipulation lead to the cosine subtraction formula by itself. This approach provides little insight into the connected and linear structure and origins of both addition formulas. It is hardly surprising that many students fail to understand these formulas. Through no fault of their own, they may feel that math is a pointless, unmotivated, and unpleasant exercise in gymnastic memorization.

Formula (1) wants and contains the Pythagorean formula that relates the horizontal and vertical components of a reference point to which $\langle 1,0\rangle$ can be rotated. Because if $\langle 1,0\rangle$ is rotated to $\langle X, Y\rangle$, then $\langle X,-Y\rangle$ returns to $\langle 1,0\rangle=\langle x X-y(-Y), x(-Y)+$ $y X\rangle=\left\langle X^{2}+Y^{2}, 0\right\rangle$. At the basic level, this is just reflection symmetry, or Pythagoras as a special case of the addition formula: $\cos (t-t)=\cos (0)=$ $1=\cos (t) \cos (-t)-\sin (t) \sin (-t)$. When scaling is incorporated, the same analysis explains the two scaling factors of $R$ in $X^{2}+Y^{2}=R^{2}$.

In 1799, Wessel saw that (1) could be interpreted to define a complex multiplication isomorphic to $(x+y i)(X+Y i)=(x X-y Y)+(y X+x Y) i$. It is no coincidence that, in the same paper, Wessel also first introduced the geometric interpretation of vector addition as uniting directed segments "in such a way that the second begins where the first ends. The sum is from the first to the last point of the united segments." Interpreting complex powers as iterated rotation and scaling permits a more complete connection among the cornerstones of the "College Algebra" curriculum: exponentials, polynomials and their zeros, and systems of linear equations. The Fibonacci-like dynamical systems that evolve deterministically from two initial conditions are a wonderful example that combines all this and more, and also leads naturally to the

\footnotetext{
${ }^{1}$ See http://math.utah.edu/\%7Epalais/AMissingPiece for this and other comparisons and examples.
}

corresponding material at the calculus level. For example, the solutions of $F_{n+2}=5 F_{n+1}-6 F_{n}$ are $c_{1} 2^{n}+c_{2} 3^{n}$, a superposition of exponentials whose bases are solutions of the polynomial equation $x^{2}=5 x-6$, and whose coefficients are found by solving a system of linear equations to match the initial conditions. When the difference equation is replaced with $y^{\prime \prime}=5 y^{\prime}-6 y$, we just change to rates for natural exponentials: $c_{1} e^{2 t}+c_{2} e^{3 t}$. Even closer to Fibonacci, when the sum is replaced by a difference, $F_{n+2}=F_{n+1}-F_{n}$, the rotation formula explains the 6-step periodicity of the basic solutions $x_{n}=2,1,-1,-2,-1,1,2,1, \ldots$ and $y_{n}=$ $0,1,1,0,-1,-1,0,1, \ldots$ They also may be written $c_{1} r_{+}^{n}+c_{2} r_{-}^{n}$, where $r_{ \pm}$are solutions of $x^{2}=x-1$, each one-sixth turn from 1 on the unit circle. The points $\left(x_{n}, y_{n}\right)$ lie on an ellipse, $x_{n}^{2}+3 y_{n}^{2}=4$, corresponding to the hyperbolas $y_{n}^{2}-5 x_{n}^{2}= \pm 4$ that contain the standard Fibonacci-Lucas pairs $x_{n}=$ $1,1,2,3,5, \ldots, y_{n}=1,3,4,7,11, \ldots$ The solutions of $y^{\prime \prime}=y-1$ are $c_{1} e^{r_{+} t}+c_{2} e^{r_{-} t}$. See the footnote link for how the rotation formula can also simplify the derivation and analysis of conic sections.

Rectangular coordinates are specified using Euclid's perpendicular bisector construction by a choice of origin, a second point for direction and unit on the first axis, and an orientation for the positive direction of the second (counterclockwise in math, clockwise in computer graphics). Then any two rectangular coordinate systems may be related by a combination of shift of origin, scaling, rotation, and reflection, corresponding to complex addition, multiplication, and conjugation, respectively! It seems a shame for our students to be deprived of the one missing transformation, rotation, when providing access to it is so elementary and permits so much utility and insight.

At the calculus level, the quarter-turn rotation formula $\langle-y, x\rangle$ also expresses the physics of uniform circular motion and Hooke's spring. When motion is neither inward nor outward, velocity must be perpendicular to displacement: $\langle x, y\rangle^{\prime}=$ $\langle-y, x\rangle$ or $z^{\prime}=i z$. This physically motivated and geometrically natural relationship provides a definition of the cosine and sine functions that parameterize our reference point $\langle X, Y\rangle$, and explains Euler's formula $e^{i t}=\cos t+i \sin t$. All other properties follow easily from this starting point. Applied twice, $\langle x, y\rangle^{\prime \prime}=\langle-x,-y\rangle$, or $z^{\prime \prime}=-z$, says acceleration is opposite to displacement. From the differential equations perspective, $\langle-y, x\rangle$ is a change of variables, the linear combination $X\langle x, y\rangle+Y\langle-y, x\rangle$ is a linear superposition of solutions, and the Pythagorean symmetry reflects their invariance under time reversal. Formula (1) is also the origin of the Cauchy-Riemann equations that characterize an analytic function of a complex variable $z$ as independent of $\bar{z}=x-i y$. 
It is well known that the negative reciprocal condition that two lines are perpendicular fails when one is vertical. The condition that two directions $\mathbf{v}=\left\langle v_{1}, v_{2}\right\rangle$ and $\mathbf{w}=\left\langle w_{1}, w_{2}\right\rangle$ are collinear, $\mathbf{w}=c \mathbf{v}$, also fails when $\mathbf{v}=\mathbf{0}$. The vanishing of the plane cross product $\mathbf{v} \times \mathbf{w}=$ $v_{1} w_{2}-v_{2} w_{1}$ is a test for collinearity that does not suffer from this exception. Directions are perpendicular if a quarter-turn rotation makes them collinear. Combining the cross product and the quarter-turn rotation formula, the vanishing of plane dot product $\mathbf{v} \cdot \mathbf{w}=v_{1} w_{1}+v_{2} w_{2}$ is a test for orthogonality that has no exception.

The plane rotation formula provides a great setting to introduce geometric vector algebra and matrices, where equation (1) takes the form $\left[\begin{array}{cc}X & -Y \\ Y & X\end{array}\right]\left[\begin{array}{l}X \\ y\end{array}\right]$. A direct calculation using plane rotation formula (1) and its consequence $X^{2}+Y^{2}=1$ confirms that, if the same rotation $\mathbf{R}$ is applied to both $\mathbf{v}$ and $\mathbf{w}$, the dot product of the images is the same: $\mathbf{R v} \cdot \mathbf{R w}=\mathbf{v} \cdot \mathbf{w}$. We may simultaneously rotate any such vectors so that the image of the first is along the positive $x_{1}$-axis, $\left\langle r_{1}, 0\right\rangle$, and that of the second is $\left\langle r_{2} \cos t, r_{2} \sin t\right\rangle$, where $r_{1}^{2}=v_{1}^{2}+v_{2}^{2}$ and $r_{2}^{2}=w_{1}^{2}+w_{2}^{2}$. This standard configuration exhibits the meaning of the dot product $\mathbf{v} \cdot \mathbf{w}=r_{1} r_{2} \cos t$, where $t$ is the angle between $\mathbf{v}$ and $\mathbf{w}$. Any rotation of space in three or higher dimensions and the standard configuration may be constructively obtained through a sequence of coordinate plane rotations, so the dot product $\mathbf{v} \cdot \mathbf{w}=\sum_{j=1}^{n} v_{j} w_{j}$ immediately inherits the same invariance and interpretation. Therefore, the generalization of the above dot product to $\mathbf{v} \cdot \mathbf{w}=\sum_{j=1}^{n} v_{j} w_{j}$ immediately inherits the same invariance and interpretation. The definition, invariance, and interpretation of the three-dimensional cross product

$$
\begin{aligned}
& \mathbf{v} \times \mathbf{w} \\
& =v_{1}\left\langle 0,-w_{3}, w_{2}\right\rangle+v_{2}\left\langle w_{3}, 0,-w_{1}\right\rangle+v_{3}\left\langle-w_{2}, w_{1}, 0\right\rangle
\end{aligned}
$$

can also be easily understood in terms of coordinate plane rotations.

Collectively, these observations give rise to a useful and purely three-dimensional interpretation of quaternions and their multiplication that we will discuss elsewhere. There are many more examples that cannot be covered here, but perhaps this brief survey will stimulate more inquiry, discussion, and discovery!

\section{Mathematical Sciences Center}

\section{Tsinghua University, Beijing, China}

\section{Positions: \\ Distinguished Professorship; Professorship; Associate Professorship; Assistant Professorship (tenure-track).}

The MSC invites applications for the above positions in the full spectrum of mathematical sciences: ranging from pure mathematics, applied PDE, computational mathematics to statistics. The current annual salary range is between $0.15-1.0$ million RMB. Salary will be determined by applicants' qualification. Strong promise/track record in research and teaching are required. Completed applications must be electronically submitted, and must contain curriculum vitae, research statement, teaching statement, selected reprints and /or preprints, three reference letters on academic research and one reference letter on teaching, sent electronically to msc-recruitment@math.tsinghua.edu.cn

The review process starts in December 2013, and closes by April 30, 2014. Applicants are encouraged to submit their applications before February 28, 2014.

*****************************************************

\section{Positions: post-doctorate fellowship}

Mathematical Sciences Center (MSC) will hire a substantial number of post-doctorate fellows in the full spectrum of mathematical sciences. New and recent PhDs are encouraged for this position.

A typical appointment for post-doctorate fellowship of MSC is for three-years.Salary and compensation package are determined by qualification, accomplishment, and experience. MSC offers very competitive packages.

Completed applications must contain curriculum vitae, research statement, teaching statement, selected reprints and /or preprints, three reference letters, sent electronically to msc-recruitment@math.tsinghua.edu.cn

The review process starts in December 2013, and closes by April 30, 2014. Applicants are encouraged to submit their applications before February 28, 2014. 